\title{
MAXIMAL CONVERGENCE GROUPS AND RANK ONE SYMMETRIC SPACES
}

\author{
ARA BASMAJIAN ${ }^{\varpi}$ and MAHMOUD ZEINALIAN
}

(Received 12 February 2005; revised 16 March 2006)

Communicated by C. D. Hodson

\begin{abstract}
We show that the group of conformal homeomorphisms of the boundary of a rank one symmetric space (except the hyperbolic plane) of noncompact type acts as a maximal convergence group. Moreover, we show that any family of uniformly quasiconformal homeomorphisms has the convergence property. Our theorems generalize results of Gehring and Martin in the real hyperbolic case for Möbius groups. As a consequence, this shows that the maximal convergence subgroups of the group of self homeomorphisms of the $d$-sphere are not unique up to conjugacy. Finally, we discuss some implications of maximality.
\end{abstract}

2000 Mathematics subject classification: primary 30F40; secondary 30G65, 37i:30.

\section{Introduction}

The convergence property (Definition 2.1) is an essential property that all families of Möbius transformations possess [2]. Many of the basic theorems in the theory of Kleinian groups can be proven within this topological context. Quasiconformal and convergence families have been studied in various contexts, see, for example, [2] and [11]. In [2], Gehring and Martin showed that, for $d \geq 2$, the Möbius group acting on the $d$-sphere is maximal in the sense that it is a convergence group and that no group of homeomorphisms of the boundary sphere having the convergence property can properly contain it. Since the isometry groups of rank one symmetric spaces of noncompact type are natural generalizations of the Möbius groups, it is of interest to see if these groups possess the same convergence and maximality property. We show the following.

THEOREM 3.4. Let $\mathbb{H}$ be a rank one symmetric space of noncompact type, which is not the real hyperbolic plane. Then the group of conformal homeomorphisms of $\partial \mathrm{QD}$ is

(C) 2007 Australian Mathematical Society 1446-7887/07 \$A2.00+0.00 
a convergence group. In fact, any family $\mathscr{F}$ of $K$-quasiconformal homeomorphisms of $\mathrm{\partial}$ ㅇt has the convergence property.

For real hyperbolic space of dimension greater than two, the above theorem was proven by Gehring and Martin in [2]. Our proof of the general case (for all rank one symmetric spaces of noncompact type) applied to real hyperbolic space is significantly different than theirs. See Tukia [11] for related results.

THEOREM 3.5. Let $\mathbb{H}_{\mathbb{C}}$ denote complex hyperbolic space of complex dimension greater than one. Then the group of conformal homeomorphism of its boundary, $\operatorname{Conf}\left(\partial \mathbb{H}_{\mathbb{C}}\right)$, is a maximal convergence group. That is,

(1) $\operatorname{Conf}\left(\partial \mathbb{H}_{\mathbb{C}}\right)$ is a convergence group, and

(2) If $G \supseteq \operatorname{Conf}\left(\partial \mathrm{H}_{\mathbb{C}}\right)$ is a convergence group acting on $\partial \mathrm{H}_{\mathbb{C}}$, then $G=\operatorname{Conf}\left(\partial \mathrm{H}_{\mathbb{C}}\right)$.

Putting Theorem 3.5 together with the results of Gehring, Martin [2] and Pansu [10], as a corollary we have the following.

COROLLARY 3.6. Let $\mathbb{\text { HO }}$ be a rank one symmetric space of noncompact type different from the real hyperbolic plane. Then the group of conformal homeomorphisms, $\operatorname{Conf}(\partial \mathbb{H})$, is a maximal convergence group.

This shows that the maximal convergence subgroups of the group of self homeomorphisms of the $d$-sphere, Homeo $\left(S^{d}\right)$, are not unique up to conjugacy. In fact, for infinitely many values of $d$, the topological group $\operatorname{Homeo}\left(S^{d}\right)$ contains maximal convergence subgroups of different dimensions. For example, the ten dimensional $\operatorname{Isom}\left(\mathbb{G}_{\mathbb{R}}^{4}\right)$ and the eight dimensional Isom $\left(\mathbb{H}_{\mathbb{C}}^{4}\right)$ act on the 3 -sphere as maximal convergence groups.

In Section 4, we mention some elementary consequences of Corollary 3.6 to convergence groups and quasi-isometry groups acting on $\mathbb{H} \cup \partial \mathfrak{H}$.

The reason for excluding the hyperbolic plane from the above discussion is that unlike the higher dimensional situation, the hyperbolic plane has trivial conformal structure on the boundary. For instance, all smooth diffeomorphisms of the circle are conformal. In this case, the affine structure of the boundary and quasisymmetric mappings may be utilized.

Finally, in the literature some authors use the term convergence group to mean a discrete convergence group. Such a restriction is not imposed in this paper.

\section{Basics}

Cartan's classification of semisimple Lie groups implies that there are three families of rank one symmetric spaces of noncompact type and an exceptional one: real 
hyperbolic space $\mathbb{V}_{\mathbb{R}}^{n}$, complex hyperbolic spaces $\mathbb{U}_{\mathbb{C}}^{2 n}$, quaternionic hyperbolic space $\mathbb{H}_{\mathbb{H}}^{4 n}$, and the Cayley plane $\mathbb{H}_{C a}^{16}$ (see [7]). Throughout this paper $\mathbb{H}$ stands for a rank one symmetric space of noncompact type except the hyperbolic plane (or equivalently complex hyperbolic space of complex dimension one). The boundary of $\mathbb{A}-1$, denoted by $\partial \mathbb{H}$, is a smooth sphere, which is naturally endowed with a conformal structure. Isom $(\mathbb{H})$ denotes the isometry group of $\mathbb{N}$ and $\operatorname{Conf}(\partial \mathbb{H})$ stands for the group of conformal homeomorphisms of the ideal boundary. Isometries of $\mathbb{t}$ induce conformal homeomorphisms on $\partial \mathbb{H}$. That is, the image of the natural map $\phi: \operatorname{Isom}(\mathbb{H}) \rightarrow$

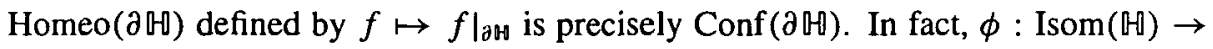
$\operatorname{Conf}(\partial \mathbb{H})$ is an isomorphism. The stabilizer of a point $x \in \mathbb{H}$ is a maximal compact subgroup of Isom( $(\mathbb{E})$, which we denote by $\mathscr{K}$. The Iwasawa decomposition says Isom(代) $=\mathscr{K} \mathscr{A} \mathscr{N}$; where $\mathscr{A}$ is a one-parameter group of translations along the geodesic connecting $x$ to $\infty$, and $\mathscr{N}$ is a nilpotent group (see [8, Section 29]). Each such $\mathscr{N}$ has a fixed point $\infty \in \partial \mathbb{H}$, and acts simply transitively on $\partial \mathbb{A}-\{\infty\}$. Thus, the ideal boundary, $\partial \Vdash$, can be identified with the one point compactification of $\mathscr{N}$, where $\mathscr{N}$ has a naturally defined (Euclidean metric in the real case) CarnotCaratheodory metric, denoted $d_{c}$, on it. With respect to this metric, the elements of $\mathscr{N}$ act as isometries, and the elements of $\mathscr{A}$ as dilations. Thus associated to each $g \in \mathscr{A}$ is a positive real number (its dilation factor) $\lambda(g)$ satisfying, $d_{c}(g(x), g(y))=$ $\lambda(g) d_{c}(x, y)$, for all $x, y \in \mathscr{N}$. Finally, the group of conformal homeomorphisms of $\partial \mathrm{H}$ acts almost triply transitively on points. That is, given three distinct points $x, y, z \in \partial \mathbb{H}$, and a positive real number $r$, there exists an element of $\operatorname{Conf}(\partial \mathbb{H})$ that takes $x$ to the identity element $e \in \mathscr{N}, y$ to $\infty$, and $z$ to the Carnot-Caratheodory sphere of radius $r$ centered at $e \in \mathscr{N}$ or any other fixed sphere. This fact, follows from the above description of the actions of $\mathscr{N}$ and $\mathscr{A}$ on $\mathbb{H}$. For a discussion on some of the basics of the complex case, see [1] and [6].

Throughout this paper all sequences are assumed to be infinite. As a matter of convention, a subsequence is often denoted with the same notation as the original sequence. We use $\rho(\cdot, \cdot)$ to denote the spherical distance on $S^{d}$.

Definition 2.1. Let $Y$ be a compact topological space. A family $\mathscr{F} \subset \operatorname{Homeo}(Y)$ is said to have the convergence property if each infinite sequence $\left\{f_{n}\right\}$ of $\mathscr{F}$ contains a subsequence, which:

(C1) converges uniformly to an element of $\operatorname{Homeo}(Y)$, or

(C2) has the attractor-repeller property, that is, there exists a point $a \in Y$, the attractor, and a point $r \in Y$, the repeller, so that the $\left\{f_{n}\right\}$ converge to the constant function $a$, uniformly outside of any open neighborhood of $r$. Note that $a$ may equal $r$.

REMARK 2.2. If the sequence $\left\{f_{n}\right\}$ uniformly converges to $f$, then $\left\{f_{n}^{-1}\right\}$ uniformly converges to $f^{-1}$. Similarly, if $\left\{f_{n}\right\}$ has attractor $a$ and repeller $r$, then $\left\{f_{n}^{-1}\right\}$ has 
attractor $r$ and repeller $a$.

Discreteness for a convergence group is equivalent to any infinite sequence having a subsequence for which axiom (C2) holds.

Definition 2.3. Let $(X, d)$ be a metric measure space. For a map $f: X \rightarrow X$, $x \in X$, and $r>0$ define

$$
\begin{aligned}
L_{f}(x, r) & =\max \{d(f(x), f(y)) \mid d(x, y)=r\}, \quad H_{f}(x, r)=\frac{L_{f}(x, r)}{l_{f}(x, r)}, \\
l_{f}(x, r) & =\min \{d(f(x), f(y)) \mid d(x, y)=r\}, \quad H_{f}(x)=\limsup _{r \rightarrow 0} H_{f}(x, r) .
\end{aligned}
$$

A homeomorphism $f$ is called quasiconformal, if $H_{f}(x)$ is uniformly bounded. In the presence of a measure, we say $f$ is $K$-quasiconformal, if it is quasiconformal and for almost every $x \in X, H_{f}(x) \leq K$. The map $f$ is said to be $K^{\prime}$-quasisymmetric if $H_{f}(x, r) \leq K^{\prime}$ for all $x \in X$ and all $r>0$. A family is called uniformly $K$ quasiconformal or uniformly $K^{\prime}$-quasisymmetric if there are constants $K$ or $K^{\prime}$ for which all maps in the family are $K$-quasiconformal or $K^{\prime}$-quasisymmetric, respectively.

The above definition of a quasiconformal map is usually referred to as the metric definition. It follows from [4, Theorem 10.19], that a homeomorphism $f: \mathscr{N} \rightarrow \mathscr{N}$ is $K^{\prime}$-quasisymmetric if and only if there exists a homeomorphism $\eta:[0, \infty) \rightarrow$ $[0, \infty)$ such that $d(x, a) \leq t d(x, b)$ implies $d(f(x), f(a)) \leq \eta(t) d(f(x), f(b))$ for every $a, b, x \in X$ and for every $t \in[0, \infty)$. The homeomorphism $\eta$ and $K^{\prime}$ depend only on each other and data associated to the space.

A $K$-quasisymmetric map is always $K^{\prime}$-quasiconformal, for $K^{\prime}=K$. Conversely, for a large class of metric spaces, including the boundaries of rank one symmetric spaces of noncompact type, a $K$-quasiconformal map is $K^{\prime}$-quasisymmetric, where $K^{\prime}$ depends only on $K$ and the data associated to the ideal boundary. See [5, Lemma 4.6 and Lemma 4.8] for details. This implies that on these metric spaces a uniformly $K$-quasiconformal family is also uniformly $K^{\prime}$-quasisymmetric.

\section{Maximality of conformal groups}

The following lemma allows us to make several simplifications, through normalization, in our arguments involving the convergence property.

LEMMA 3.1. Suppose $\left\{g_{n}: \partial \mathbb{H} \rightarrow \partial \mathbb{H}\right\}$ and $\left\{h_{n}: \partial \mathbb{H} \rightarrow \partial \mathbb{H}\right\}$ uniformly converge to the self homeomorphisms $g: \partial \mathbb{H} \rightarrow \partial \mathrm{H}$ and $h: \partial \mathrm{H} \rightarrow \partial \mathrm{M}$, respectively. Then the sequence $\left\{f_{n}\right\}$ satisfies axiom (C1) or (C2) if and only if $\left\{g_{n} f_{n} h_{n}\right\}$ satisfies $(\mathrm{C} 1)$ or (C2), respectively. 
ProOF. The (C1) axiom equivalence follows from the general properties of topological groups. For the (C2) equivalence, it is enough to show the property first for postcomposition by $g_{n}$ and then for precomposition by $h_{n}$. To this end, suppose $\left\{f_{n}\right\}$ locally uniformly converges to $a$ on $\partial H-\{r\}$. Recall that the spherical metric on $\partial H$ is denoted $\rho(\cdot, \cdot)$. Let $C$ be a compact set in $\partial \mathbb{H}-\{r\}$ and let $\epsilon>0$. Choose $\delta>0$ such that $g$ maps the $\rho$-ball of radius $\delta$ centered at $a$ into the $\rho$-ball of radius $\epsilon / 2$ around $g(a)$. Choose $n$ sufficiently large so that $\rho\left(g_{n}(x), g(x)\right)<\epsilon / 2$, for all $x \in \partial \mapsto$, and that $f_{n}(C)$ lands in the $\rho$-ball of radius $\delta$ centered at $a$. Clearly $g_{n} f_{n}(C)$ is in the $\rho$-ball of radius $\epsilon$ about $g(a)$ for large enough $n$. Thus $\left\{g_{n} f_{n}\right\}$ locally uniformly converges to $g(a)$ in $\partial \mathbb{H}-\{r\}$. The converse follows from group properties. We have proven the lemma for postcomposition.

To prove the lemma for precomposition, write $f_{n} h_{n}$ as $\left(h_{n}^{-1} f_{n}^{-1}\right)^{-1}$ and use $\operatorname{Re}$ mark 2.2.

LEMMA 3.2. Let $\left\{f_{n}: \partial \mathbb{H} \rightarrow \partial \mathbb{N}\right\}$ be a sequence of $K$-quasiconformal mappings. There exist $g_{n}: \partial \mathbb{H} \rightarrow \partial \mathbb{H}$ in Conf( $(\partial \mathbb{H})$, where $g_{n}\left(f_{n}(\infty)\right)=\infty$ and $g_{n} f_{n}$ is $K$ quasiconformal. Moreover, a subsequence of $\left\{f_{n}\right\}$ satisfies $(\mathrm{C} 1)$ or $(\mathrm{C} 2)$ if and only if a subsequence of $\left\{g_{n} f_{n}\right\}$ satisfies $(\mathrm{C} 1)$ or $(\mathrm{C} 2)$, respectively.

PROOF. Since $\mathscr{K}$ is compact and acts transitively on $\partial \mathscr{H}$, we can find $g_{n} \in \mathscr{K}$ so that $g_{n} f_{n}(\infty)=\infty$, and $g_{n}$ uniformly converges to $g \in \mathscr{K}$. Hence the first part of the lemma follows because the elements of $\mathscr{K}$ are conformal. The last statement follows from Lemma 3.1.

We omit the proof of the following lemma, since it closely follows that of the real case given in [2].

LEMMA 3.3. Let $\mathscr{F} \subset$ Homeo(JH) be a family of self homeomorphisms. Furthermore, assume that $\mathscr{F}$ is closed under post and precomposition by $\operatorname{Conf}(\partial \mathrm{H})$. If $\mathscr{F}$ has the convergence property then $\mathscr{F}$ is a uniformly quasiconformal family with respect to the conformal structure on $\partial \mathrm{H}$.

Using the above lemmas, we have the following result.

THEOREM 3.4. Let $\mathbb{H}$ be a rank one symmetric space of noncompact type which is not the real hyperbolic plane. Then the group of conformal homeomorphisms of $2 \mathbb{W}$ is a convergence group. In fact, any family $\mathscr{F}$ of $K$-quasiconformal homeomorphisms of $\partial$ OT has the convergence property.

PROOF. To show that the conformal group is a convergence group, set $\partial \mathrm{ND}=$

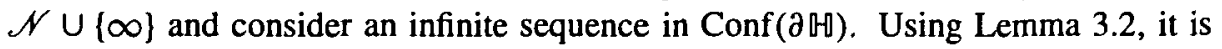
enough to show that a sequence $\left\{g_{n}\right\}$, which fixes $\infty$ has an infinite subsequence that 
satisfies (C1) or (C2). As remarked in the basics section, an element of Conf $(\partial \mapsto H)$, which fixes $\infty$ acts as a similarity with respect to the (Euclidean in the real case) Carnot-Caratheodory metric, $d_{c}$, on $\mathscr{N}$. Next, by possibly passing to a subsequence and inverses, we may assume that the $\left\{g_{n}\right\}$ are distance nondecreasing on $\mathscr{N}$. We continue to call the subsequence $\left\{g_{n}\right\}$. According to the Iwasawa decomposition, $g_{n} \in \mathscr{K} \mathscr{A} \mathscr{N} ;$ more precisely, $g_{n}=R_{n} \Lambda_{n} T_{n}$, where $R_{n} \in \mathscr{K}$ fixes $\infty, \Lambda_{n}$ is a dilation with expansion factor $\lambda_{n} \geq 1$, and $T_{n} \in \mathscr{N}$. Since $\mathscr{K}$ is compact, again by passing to a subsequence, we may assume $\left\{R_{n}\right\}$ uniformly converges to $R \in \mathscr{K}$ and hence by Lemma 3.1, it is enough to consider the sequence $g_{n}=\Lambda_{n} T_{n}$.

If $\left\{T_{n}\right\}$ has a convergent subsequence then using Lemma 3.1, it is enough to consider the sequence of dilations $\left\{\Lambda_{n}\right\}$. However, such a sequence clearly has the convergence property.

Otherwise, if $\left\{T_{n}\right\}$ does not have a convergent subsequence, then $\left\{T_{n}\right\}$ locally uniformly converges to $\infty$ in $\partial H-\{\infty\}$. Since $\lambda_{n} \geq 1$, the sequence $\left\{\Lambda_{n} T_{n}\right\}$ shares the same convergence property.

In order to show that any uniformly quasiconformal family $\mathscr{F}$ has the convergence

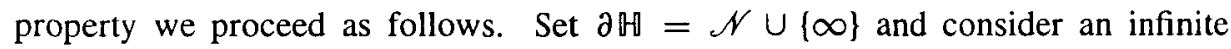
sequence $\left\{f_{n}\right\} \subset \mathscr{F}$. Using Lemma 3.2 , it is enough to show that a sequence $\left\{f_{n}\right\}$ of $K$-quasiconformal homeomorphisms of $\partial H$ which fix $\infty$ have an infinite subsequence which satisfies (C1) or (C2). Post compose each $f_{n}$ by an element $T_{n} \in \mathscr{N}$ which takes $f_{n}(e)$ to $e$. Next, fix $p \in \partial \mathbb{H}-\{e, \infty\}$ and choose an element $\Lambda_{n} \in \mathscr{A}$ taking $T_{n} f_{n}(p)$ to the sphere of radius one centered at the origin.

Set $g_{n}=\Lambda_{n} T_{n}$ and consider the sequence $\left\{g_{n} f_{n}\right\}$. The $g_{n} f_{n}$ fix $e$ and $\infty$ as well as taking $p$ to the unit sphere centered at the origin. Now by [5. Theorem 4.8], $\left\{g_{n} f_{n}\right\}$ being $K$-quasiconformal implies $\left\{g_{n} f_{n}\right\}$ is $K^{\prime}$-quasisymmetric on $\mathscr{N}$, where $K^{\prime}$ only depends on $K$. Therefore, by [4, Theorem 10.26], $\left\{g_{n} f_{n}\right\}$ has a subsequence (we continue to use the same notation for the subsequence), which converges locally uniformly on $\mathscr{N}$ to a map, say $h$. By [4, Exercise 10.29], $h$ is either a $K^{\prime}$-quasisymmetric embedding or is constant on $\mathscr{N}$. Since $g_{n} f_{n}(p)$ is in the sphere of radius one, $h$ is not constant. We need to show that $h: \mathscr{N} \rightarrow \mathscr{N}$ is surjective.

To this end, consider $\mathscr{N}^{\prime}=\partial \mathbb{H}-\{e\}$. Again $\left\{g_{n} f_{n}\right\}$ being $K$-quasiconformal implies $\left\{g_{n} f_{n}\right\}$ is $K^{\prime}$-quasisymmetric on $\mathscr{N}^{\prime}$. The above argument implies $\left\{g_{n} f_{n}\right\}$ locally uniformly converges to an embedding $h^{\prime}$ of $\mathscr{N}^{\prime}$. Clearly $h=h^{\prime}$ on $\partial \square-\{e, \infty\}$ and therefore $h$ is surjective. Thus, the sequence $\left\{g_{n} f_{n}\right\}$ uniformly converges to the self homeomorphism $h$ of $\partial \leftrightarrow$.

Writing $f_{n}$ as $g_{n}^{-1}\left(g_{n} f_{n}\right)$ and appealing to Lemma 3.1, we see that $\left\{f_{n}\right\}$ satisfies (C1) or (C2) if and only if $\left\{g_{n}^{-1}\right\}$ does. However, the elements of $\left\{g_{n}^{-1}\right\}$ are in Conf $(\partial \mathbb{H})$ which we have shown to have the convergence property.

THEOREM 3.5. Let $\mathbb{H}_{\mathbb{C}}$ denote complex hyperbolic space of complex dimension 
greater than one. Then the group of conformal homeomorphism of its boundary, $\operatorname{Conf}\left(\partial \mathbb{H}_{\mathbb{C}}\right)$, is a maximal convergence group. That is,

(1) $\operatorname{Conf}\left(\partial \mathrm{H}_{\mathbb{C}}\right)$ is a convergence group, and

(2) If $G \supseteq \operatorname{Conf}\left(\partial \mathbb{Q}_{\mathbb{C}}\right)$ is a convergence group acting on $\partial \mathbb{H}_{\mathbb{C}}$, then $G=\operatorname{Conf}\left(\partial \mathrm{H}_{\mathbb{C}}\right)$.

PROOF. The first item was proven in Theorem 3.4. For the maximality, suppose $f \in G$ is not conformal. Then by [9, Lemma 18.5], $f$ is not globally 1-quasiconformal using the metric definition. That is, the dilatation $H_{f}(x)>1$ on a set of positive measure.

The map $f$ is almost everywhere Pansu differentiable (see [9, Théorème 5]). Hence there is a point of differentiability, which we may assume to be $e \in \partial \mapsto \mathbb{N}=\mathscr{N} \cup \infty$ satisfying $H_{f}(e)>1$. Using [6, Proposition 7] we have,

$$
\lambda_{1}^{2 n+2}(e) \leq K^{n+1} J_{f}(e) .
$$

Here $\lambda_{1}(e)$ is the length of the large axis in the image of the unit sphere in the contact subspace at $e$ under the ordinary derivative of $f$. Also, $J_{f}(e)$ is the determinant of the Pansu derivative at $e$.

Postcomposing by an element of $\operatorname{Conf}\left(\partial \mathbb{H}_{\mathbb{C}}\right)$, we may assume that $f(e)=e$ and $J_{f}(e)$ is one. Moreover, since the stabilizer of $e$ in Conf $\left(\partial \mathrm{H}_{C}\right)$ acts transitively on directions in the contact subspace at $e$, we may assume that $\lambda_{1}(e)$ is an eigenvalue of $f$ with eigenvector in the contact subspace. By the chain rule all iterates of $f$ have $e$ as a point of Pansu differentiability. Thus by inequality (3.1), iterating $f$ gives rise to quasiconformal maps with arbitrarily large dilatation in $G$, which is a contradiction. Therefore, $G=\operatorname{Conf}\left(\partial \mathrm{H}_{\mathbb{C}}\right)$.

For real hyperbolic space of dimension greater than two, the fact that the Möbius group is a maximal convergence group acting on $\partial \mathrm{H}-\mathrm{O}$ was proven in Gehring-Martin [2]. In the case of Quaternionic or Cayley Hyperbolic space, all quasiconformal self homeomorphisms of $\partial \mathrm{H}_{\mathrm{H}}^{4 n}(n \geq 2)$, and $\partial \mathbb{H}_{\mathrm{Ca}}^{16}$ are 1-quasiconformal, and hence conformal. This follows from [10, (11.2) and (11.5)]. Putting these results together with Theorem 3.5 we have the following.

COROLlARY 3.6. Let $\mathbb{H}$ be a rank one symmetric space of noncompact type different from the real hyperbolic plane. Then the group of conformal homeomorphisms, $\operatorname{Conf}(\partial \mathrm{H})$, is a maximal convergence group.

\section{Some consequences of maximality}

We remind the reader that $\mathrm{OH}$ denotes a rank one symmetric space of noncompact type except for the hyperbolic plane. The following fact is an immediate application of Corollary 3.6. 
COROLLARY 4.1. The group of conformal homeomorphisms, Conf(วR), is a maximal uniformly quasiconformal group.

Let $\mathrm{QC}(\partial \mathbb{H})$ denote the group of quasiconformal homeomorphisms of the boundary. A full quasi-isometry of $\mathbb{H}$ is a quasi-isometry $f: \mathbb{H} \rightarrow \mathbb{H}$ whose domain and image is $\mathbb{H}$; two such are equivalent if the Hausdorff distance between their graphs in $\mathbb{H} \times \mathbb{H}$ is finite. Let QI $(\mathrm{H})$ denote the group of equivalence classes of full quasi-isometries of $\mathbb{0}$. Note that Isom $(\mathbb{H})$ naturally embeds in QI(H) $)$. A family $\mathscr{F} \subset \mathrm{QI}(\mathbb{H})$ is said to be a uniformly quasi-isometric family if there exists a uniform bound on the Lipschitz constant for all elements of $\mathscr{F}$. It is well known that a quasi-isometry class $[f]$ induces a quasiconformal homeomorphism, $\left.f\right|_{\partial H}$, on the boundary. Moreover, the quasiconformality constant is dependent solely on the Lipschitz constant of the quasiisometry (see [10]). In fact, the map $\left.[f] \mapsto f\right|_{\partial H}$ is a group isomorphism between $\mathrm{QI}(\mathbb{H})$ and $\mathrm{QC}(\partial \mathbb{H})$, which identifies $\operatorname{Isom}(\mathbb{H})$ with $\operatorname{Conf}(\partial \mathbb{Q})$. Moreover, $\mathbb{B O}_{\mathbb{H}}^{4 n}$, for $n \geq 2$, and $\mathbb{H}_{\mathbb{C} a}^{16}$ are QI-rigid; that is, $\operatorname{Isom}\left(\mathbb{H}_{M}^{4 n}\right)=\mathrm{QI}\left(\mathbb{H}_{\forall-1}^{4 n}\right)$ and $\operatorname{Isom}\left(\mathbb{H}_{\mathbb{C} a}^{16}\right)=$

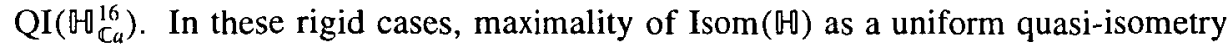
group is a triviality. A more interesting phenomenon is that of the maximality of Isom $(\mathbb{N})$ in the remaining cases of $\mathbb{H}_{\mathbb{R}}^{n}$, for $n \geq 3$, and $\mathbb{H}_{\mathbb{C}}^{2 n}$, for $n \geq 2$. This fact, which has been observed in Gromov and Pansu [3] may be thought of as a simple consequence of Corollary 4.1 .

COROLLARY 4.2. The group of isometries, Isom(N), is a maximal uniformly quasiisometric group.

Another elementary consequence of Corollary 3.6 is the following,

COROLLARY 4.3. Let $G$ be a convergence group acting on $\mathbb{H} \cup$ dه. Suppose that each element of $G$ is topologically conjugate to an element of $\operatorname{Isom}(\mathbb{W})$. If Isom $(\mathbb{N}) \leq G$, then $G=\operatorname{Isom}(\mathbb{H})$. The conjugating homeomorphism need not be the same for all elements of $G$.

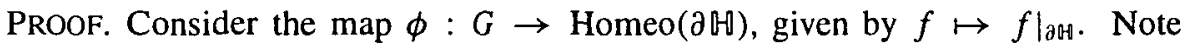
that Image $(\phi)$ is a convergence group acting on $\partial \mathbb{H}$ and contains Conf $(\partial \mathbb{H})$. Since $\operatorname{Conf}(\partial \mathrm{HA})$ is a maximal convergence group (Corollary 3.6) we have Image $(\phi)=$ $\operatorname{Conf}(\partial \mathbb{H})$.

Now take $f \in \operatorname{Ker}(\phi)$. By our assumption, there exists a homeomorphism $h$ :

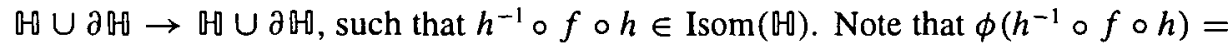
$\left.\left.\left.h^{-1}\right|_{\partial H} \circ f\right|_{\partial M} \circ h\right|_{\partial H}=\left.\left.\left.h^{-1}\right|_{\partial M} \circ \operatorname{Id}\right|_{\partial H} \circ h\right|_{\partial M}=\left.\operatorname{Id}\right|_{\partial H}$. Since $\phi$ restricted to $\operatorname{Isom}(H)$ is a monomorphism, it must be that $h^{-1} \circ f \circ h=\operatorname{Id}_{\mathrm{H}}$ and hence $f=I d_{\mathrm{H}}$; that is, $\phi: G \rightarrow \operatorname{Homeo}(\partial \mathbb{N})$ is a monomorphism. Since Image $(\phi)=\phi(\operatorname{Isom}(\mathbb{N}))=$ $\operatorname{Conf}(\partial \mathbb{H})$, we may conclude $G=\operatorname{Isom}(\mathbb{H})$. 


\section{References}

[1] A. Basmajian and R. Miner, 'Discrete subgroups of complex hyperbolic motions', Invent. Math. 131 (1998), 85-136.

[2] F. W. Gehring and G. J. Martin, 'Discrete quasiconformal groups I', Proc. London Math. Soc. (3) 55 (1987), 331-358.

[3] M. Gromov and P. Pansu, Rigidity of lattices: an introduction, Lecture Notes in Math. 1504 (Springer, Berlin, 1991) pp. 39-137.

[4] J. Heinonen, Lectures on analysis on metric spaces, Universitext (Springer-Verlag, New York, 2001).

[5] J. Heinonen and P. Koskela, 'Quasiconformal maps in metric spaces with controlled geometry', Acta Math. 181 (1998), 1-61.

[6] A. Koranyi and H. M. Reimann, 'Foundations for the theory of quasiconformal mappings on the Heisenberg group', Adv. Math. 111 (1995), 1-87.

[7] G. Mostow, Strong rigidity of locally symmetric spaces, Ann. Math. Stud. 78 (Princeton University Press, Princeton, 1978).

[8] P. Pansu, Quasiconformal mappings and manifolds of negative curvature, Lecture Notes in Math. 1201 (Springer, Berlin, 1986).

[9] _-, Quasiisometries des varietes a courbure negative ( $\mathrm{Ph} . \mathrm{D}$. Thesis, Paris, 1987).

[10] — 'Metrique de Carnot-Carathéodory et quasi-isométries des espaces symmetrique de rang un', Ann. of Math. (2) 129 (1989), 1-60.

[11] P. Tukia, 'Convergence groups and Gromov's metric hyperbolic spaces', New Zealand J. Math. 23 (1994), 157-187.

Department of Mathematics

Hunter College and Graduate Center

City University of New York

365 Fifth Avenue

New York NY 10016-4309

USA

e-mail: abasmaji@hunter.cuny.edu
Department of Mathematics

C.W. Post Campus

Long Island University 720 Northern Boulevard

Brookville, NY 11548

USA

e-mail:mzeinalian@liu.edu 
